State of the art

David Bailin

Electroweak Interactions. An Introduction to the Physics of Quarks and Leptons. By Peter Renton. Cambridge University Press: 1990. Pp. 596. £60; \$110.

QUANTUM electrodynamics (QED) is a relativistic quantum field theory formulated by Feynman, Dyson and Schwinger around forty years ago. Its incredible success in describing and predicting purely electromagnetic processes with apparently limitless accuracy in terms of very few parameters, naturally suggested that the other interactions of particle physics, the weak and strong interactions, should also be described by relativistic quantum field theories. We now know that this expectation is correct, and that the weak and electromagnetic interactions are described by a unified gauge field theory called electroweak theory, while the strong interactions are described by quantum chromodynamics (QCD).

Electroweak theory differs from QED in several important respects. First, the gauge symmetry group $(\mathrm{SU}(2) \times \mathrm{U}(1))$ underlying the theory is larger than the simple U(1) group (of transformations on a circle) which underlies QED. Second, this group is non-abelian, meaning that it is non-commutative. Finally, the theory is 'chiral', reflecting the fact that weak interactions, unlike electromagnetic ones, are not invariant under the parity transformation: we can say that left and right chiral states have different weak charges. None of these differences is much more than technical, and in principle an SU(2) $\times U(1)$ invariant gauge theory could have been formulated at any time after about 1954. The really vital difference from QED is that the symmetry is broken. If it were not, the weak gauge particles, the $\mathrm{W}$ and $\mathrm{Z}$ bosons, would be massless, just as the photon is and, because the theory is chiral, the electron, muon and other fermions would also be massless. Breaking the gauge symmetry is even easier than preserving it, but in general the breaking destroys the 'renormalizability' of the symmetric theory. In a renormalizable theory, such as QED, the theory is rendered finite by absorbing (ultraviolet) divergences into a finite number of (unpredicted) parameters. The problem with breaking the symmetry by hand, as it were, is that an infinite number of such renormalizations is needed, and the theory loses the ability to predict, which is the hallmark of its paradigm (QED). The trick, we now know, is to break the symmetry 'spontaneously'; the dynamics preserves the symmetry, but the ground state (the particle-physics vacuum) breaks it. (It was Weinberg's use of this technique on Glashow's gauge symmetric lagrangrian which finally led to the electroweak quantum field theory, whose renormalizability was demonstrated in the early 1970 s.)

Most of the components of this theory have now been tested against experiment with overwhelming success. It is the renormalizability of the theory which permitted the prediction of the $\mathrm{W}$ and $\mathrm{Z}$ boson masses to within about $1 \mathrm{GeV}^{-2}$ of the values measured when the particles were triumphantly discovered at CERN in 1983. Similarly, the theory now provides upper and lower limits on the top quark mass before its discovery. The final 'proof' that the symmetry is spontaneously broken must await the discovery of the Higgs boson, whose mass is unfortunately not predicted by the theory. The undeniable success of the theory naturally requires that graduate students of particle physics learn the theory and how it works. This demands not merely the study of quantum field theory, and QED in particular, but also the novel features of electroweak theory to which I have alluded. Increasingly it falls to experimentalists to perform the theoretical calculations against which the data are tested, and which 15 years ago were the preserve of theoreticians.

It is therefore entirely appropriate that this book on electroweak theory is by an experimentalist, in fact one who is currently engaged in precision tests of the theory at the DELPHI detector on LEP, the electron-positron collider opened at CERN six months ago. Having an experimentalist as its author, the book is naturally replete with an abundance of very impressive data detailing just how successful the model has been. The problem for the author of such a book is where to begin, and Renton goes right to the very beginning, dealing with wave equations, field operators and QED, including its renormalization, in the first third of the book. All these topics are of course well covered (in more detail) in many books, some more than 30 years old. In petroleum retailing it is well known that if a crossroads (say) has three filling stations, then this demonstrates that the location is ideal for selling petrol and that a fourth filling station will also thrive. As it is in gas stations, so it is apparently in bookselling. Renton declares in his preface that "the main emphasis is on the understanding of physical processes in terms of lowest order calculations". $\mathrm{He}$ stresses that higher order calculations are important and refers the reader to "more advanced texts" for details. I think this is a pity. The renormalization is rather complicated, as the novel features of electroweak theory require techniques which are not required in QED. Nevertheless, as the whole point of the theory is that it is renormalizable, and therefore admits precision tests, and since experimentalists are beginning to do these calculations, I think that some attempt at covering this topic should have been made, probably dispensing with the earlier material. Another theorist's gripe would be the failure to cover the 'chiral anomalies'. It is precisely the requirement of 'anomaly cancellation' which makes us so certain that the top quark must exist. Not many books discuss either of these, so perhaps my (academic's) criticism can be rebutted by the (retailer's) response that the market is not yet ready for such an innovation.

In any case, my overall judgement is very positive, and I think that the book will be well received, especially by experimentalists. It is about much more than electroweak interactions. (It includes QCD, and the possibilities beyond the standard model.) In many ways the subtitle conveys more accurately than the main title what is in the book: an (excellent) account of the current state of particle physics.

David Bailin is in the School of Mathematica and Physical Sciences, University of Sussex, Brighton BN1 9QH, UK.

\section{Culture club}

\section{R. H. A. Coutts}

Genetic Engineering of Crop Plants. Edited by G. W. Lycett and D. Grierson. Butterworths: 1990. Pp.293. £65.

IN the first chapter of this collection of papers presented at the 49th Nottingham Easter School in Agricultural Sciences, Edward Cocking illustrates the importance and the impact of plant cell and tissue culture on plant genetic engineering; most of the subsequent contributions serve only to reinforce this statement. In some cases, where researchers are dealing with plants amenable to tissue culture and which can be regenerated, such as tobacco and potato, the results are exciting. But with other, more recalcitrant species, such as some monocotyledonous plants and legumes, optimism abounds.

The scope of the book is certainly wide ranging, from coat protein-mediated protection against plant virus infection to nodulation genes of Rhizobium. In fact these chapters, respectively from the laboratories of Roger Beachy and Tony Johnston, are highpoints in the book: the assembly of useful data includes not only that obtained by the named researchers efforts but reviews that of others. The underlying enthusiasm of molecular biologists, especially through the discovery of transgenic plant technology, is evident in the crisp and stylish presentation. 